\title{
Patterns of brown rust development in eight wheat multiline components using alternate row design
}

\author{
KS Prakash *,**, PL Gautam, BP Rao \\ Hill Campus GB Pant University of Agriculture and Technology, UP, 249 199, India
}

(Received 12 February 1992; accepted 12 October 1992)

\begin{abstract}
Summary - The intergenotypic interactions for the spread and development of natural brown rust infection in components of Kalyansona multiline wheat MLKS-11 were studied using an alternate row design. The results showed no evidence of an escape mechanism due to the shielding of the susceptible components by the resistant lines during the onset of the disease. However, there were indications of barrier effects of resistant genotypes in which these genotypes, apart from being noncontributory, slowed down the disease development in susceptible host components. The intergenotypic effects between the susceptible components themselves, or with the other susceptible lines significantly increased brown rust infection. The susceptible lines were more infected by the pathogen when they were bordered by the same lines or other susceptible host genotypes showing similar reactions to a virulent race of the pathogen. The need for an allelism test for different $L r$ gene(s) using artificial inoculations or the use of field designs such as alternate row design to elicit information on interactions for disease development before constituting a multiline has been highlighted in the results.
\end{abstract}

intergenotypic interactions / multiline / brown rust / Triticum aestivum = wheat / Puccinia recondita

Résumé - Modèles de développement de la rouille brune sur huit composants d'une variété multilignée de blé, cultivés en rangs alternés. Les interactions intergénotypiques pour l'évolution et le développement de la rouille brune en conditions naturelles ont été étudiées sur 8 composants de la variété multilignées de blé MLKS-11. Le dispositif expérimental en "rangs alternés" a été utilisé pour cette étude. Les résultats ont infirmé le "mécanisme d'échappement" dû à une protection des composants sensibles par les lignes résistantes au cours de l'installation de la maladie. Les génotypes résistants ont eu un effet de barrière physique. De plus, ces lignées ne contribuant pas à la production d'inoculum, diminuent la vitesse de développement de la maladie chez les composants sensibles. Les effets intergénotypiques entre les composants sensibles eux-mêmes, soit avec les autres lignées sensibles, ont augmenté significativement l'infection par la rouille brune. Le parasite a attaqué très fortement ces groupes de lignées. Ces résultats confirment la nécessité de l'étude des gènes "Lr" présents, par des inoculations artificielles et l'utilisation du dispositif en "lignes alternées" pour obtenir des informations sur les interactions intergénotypiques pour le développement de la maladie, avant de constituer une variété multilignée.

interactions génotypiques / multilignée /Triticum aestivum = blé tendre / Puccinia recondita

\section{INTRODUCTION}

Rust diseases, particularly the brown rust caused by Puccinia recondita Rob ex Desm f sp tritici Erick et Hen pose a major threat to wheat yield stability in India. Most of the resistant breeding strategies are based on the development of pure line varieties. The resistant varieties which have thus evolved tend to become susceptible even before they are put through tests due to the change in pathogen virulence pattern. This has resulted in the development of an alternative strategy to combat the threat posed by rusts to yield stability. One such strategy is the multiline concept (Jenson, 1952; Borlaug, 1953, 1957, 1958; Browning and Frey, 1969; Clifford, 1975). It was hypothesised that multiline varieties possess synthesised horizontal resistance (Borlaug, 1958). The progression and the mode of disease development in mixed intraspecific cereal plant populations such as multiline varieties have drawn the attention of many researchers on dis-

\footnotetext{
* Correspondence and reprints

** Present address: Plant Breeding Institute, Faculty of Agriculture, The University of Sydney, NSW 2006, Australia
} 
ease epidemiology following the studies of Vanderplank (1963). Both temporal and spatial aspects of progress of the disease have been considered by various workers (Jeger et al, 1981, 1983; Mundt and Browning, 1985; Mundt and Leonard, 1985; Mundt and Brophy, 1988; Buiel et al, 1989). Among these reports, those of the Mundt group have emphasised that the susceptible host genotypic area and the number of host genotype units are the dominant factors in determining the effectiveness of multiline mixtures for disease control. Mundt and Brophy (1988) have given more weight to the number of susceptible host genotype units in the mixture based on the statistical and simulation models used by them to study the development of epidemics in mixed crop stands. Their results also suggest that the culture of alternating rows of different host genotypes in a mixture may improve the effectiveness of multilines in disease control.

However, specific intergenotypic interactions among the isogenic lines of cereal multilines for the development of infections by different races in natural epidemics have been dealt with only to a limited extent by the above studies. Further, when more components are involved in a multiline, it would add to the complexity of the problem. Information on such interactions is however, essential before constituting multilines. The nature of such integenotypic interactions operating for other characters such as plant height and grain yield among 8 isogenic components of a wheat multiline is to some extent known (Prakash et al, 1987, 1990).

Luthra and Rao (1979) have hypothesised that an 'escape' mechanism may be operating in multiline mixtures where the susceptible components may escape from the disease because of the shiedling effects of the resistant lines in proximity. Furthermore, the barrier effects as suggested by Wolfe (1985) may be provided by resistant genotypes in multiline mixtures, whereby they act as physical barriers in delaying the spread of the disease to the susceptible host genotypes. These 2 hypotheses, however, have not been supported by empirical evidence.

The present investigation was undertaken to obtain possible evidence for any of these hypotheses and to elucidate the interactions among 8 components of the multiline MLKS-11 and the susceptible recurrent parent Kalyansona. Alternate row design used by Hansen et al (1961) was chosen because of the suitability of alternate rows in a unique sequence: i) to record observations on the progress of brown rust development in each genotype laid out as a row: ii) to study the effect of the neighbour genotype rows on the development of disease in a particular host genotype; and iii) with the availability of an appropriate statistical model, to partition the variances and test their significance, thus enabling identification of the intergenotypic interactions for the disease reaction. This design has also been used by us (Prakash et al, 1987) in addition to hill plot design (Prakash et $a l, 1990)$ to obtain information on such interactions for plant height and grain yield of the components of this multiline. In this paper we have made an attempt to examine the information obtained on intergenotypic interactions associated with brown rust development among the components of this multiline.

\section{MATERIALS AND METHODS}

The experimental material comprised 8 component isogenic lines of Kalaynsona multiline MLKS-11 and the baseline Kalyansona itself. The seeds of the isolines were obtained from the project director (wheat), Indian Agricultural Research Institute, New Delhi, India. The origin and the brown rust reactions of these lines are shown in table I.

The material was planted on December 5, 1989 with a view to providing a favourable environment for the brown rust, which generally sets in around February-March. Before sowing, the experimental area was fertilised with nitrogen $(\approx 60 \mathrm{~kg} \mathrm{~N} / \mathrm{ha}$ ). The rows were $1.5 \mathrm{~m}$ long and the seed rate was maintained at $100 \mathrm{~kg} / \mathrm{ha}$. The row to row spacing was kept at $18 \mathrm{~cm}$. Good crop stand was maintained throughout the experiment. An alternate row design proposed by Hanson et al (1961) was used to determine the behaviour of each genotype planted in a row when it was bordered on either side by the same genotype and all combinations of other genotypes, eg genotype 1 could be exposed to the following $9 \times 9$ treatments:

\begin{tabular}{|c|c|}
\hline 111 & $211 \ldots . .911$ \\
\hline 112 & $212 \ldots \ldots .912$ \\
\hline 113 & $213 \ldots . .913$ \\
\hline --- & ----.......--- \\
\hline$-\cdots$ & $-\cdots . . . . . .-\cdots$ \\
\hline--- & $---\ldots \ldots .--$ \\
\hline 119 & $219 \ldots \ldots$ \\
\hline
\end{tabular}

where the central digit refers to the row of the test genotype and the adjacent digits refer to the rows of the genotypes bordering it on either side. The basic assumption made was that a combination 119 behaves in a similar manner to the reciprocal combination 911 . Hence, the number of combinations per test genotype is reduced from $p^{2}$ to $p(p+1) / 2$ where $p$ is the total number of genotypes used. Hence, for a set of 9 geno- 
Table l. Origin of experimental wheat isogenic lines and their reaction to natural brown rust infection at Pantnagar, India.

\begin{tabular}{clll} 
SINo & Genotype & Origin & General brown rust reaction at Pantnag \\
\hline & & & \\
1 & IWP-19 & E6254/KS*2 & Resistant \\
2 & IWP-72 & E6056/KS*2 & Resistant \\
3 & IWP-87-1 & E5968/KS* & Susceptible \\
4 & IWP-124 & Kalyansona cross & Resistant, necrotic spots on leaves \\
5 & IWP-127 & Frecor/KS*2 & Resistant \\
6 & IWP-129 & HS 19/KS*2 & Moderately resistant \\
7 & IWP-139 & E5863/KS*2 & Resistant \\
8 & IWP-143 & (NP875-E4894)/KS*4 & Susceptible \\
9 & Kalyansona & Pj'S" $\times$ Gabo & Highly susceptible
\end{tabular}

* KS = Kalyansona.

types there were 45 combinations. This gave a total of 405 combinations for all the 9 genotypes. Since each combination consisted of 3 rows, there were 1215 rows in each replication. To make the layout more compact, theoretically $t$ treatments can be obtained by growing $t+2$ rows. Each row acts as the test genotype as well as the competitor. Hence, given a particular unique sequence, all the 405 treatments required in the experiment can be obtained by a sequence of 407 digits. Allowing for a few unavoidable duplications, a sequence of 426 digits was generated which contained all the required 3-way permutations and combinations for the 9 genotypes. The format of this unique sequence is given in figure 1 . The randomization was restricted over replications since the sequence could in no way be disturbed. Hence, only the orientation of the sequence could be altered. The experiment was laid out in 3 replicates and the layout was bordered by infector rows consisting of a mixture of Kharchia, Agra local and Lal Bahadur, all of which were highly susceptible to natural brown rust infection in the Pantnagar environment.

The development of brown rust in the multiline components was recorded over $4 \mathrm{~d}$ of observation using a modified Cobb's scale on a linewise basis. Although the brown rust was noticed as early as late February and early March on the infector genotypes in the borders, it was not until the 16th March that the first pustules were observed on Kalyansona in the experiment. Brown rust observations were recorded using a modified Cobb's scale on 16th March, 21st March, 3rd April and 12th April respectively and the rust data recorded were normalised using arc $\sin ^{-1}$ transformation for analysis.

The following linear model as proposed by Hanson et al (1961) was used for this purpose:

$$
Y_{i k j m}=\mu+r_{i}+G_{j}+C_{k j}+C_{j m}+S_{k j m}+e_{i j m}
$$

where, $k, j$ and $m$ indicate the values from 1 to $p$ where $p$ is the total number of genotypes used; $\mu$ is the population mean and $G_{j}$ the genotypic effect. $G_{j}$ is the average phenotypic deviation for the th genotype within a

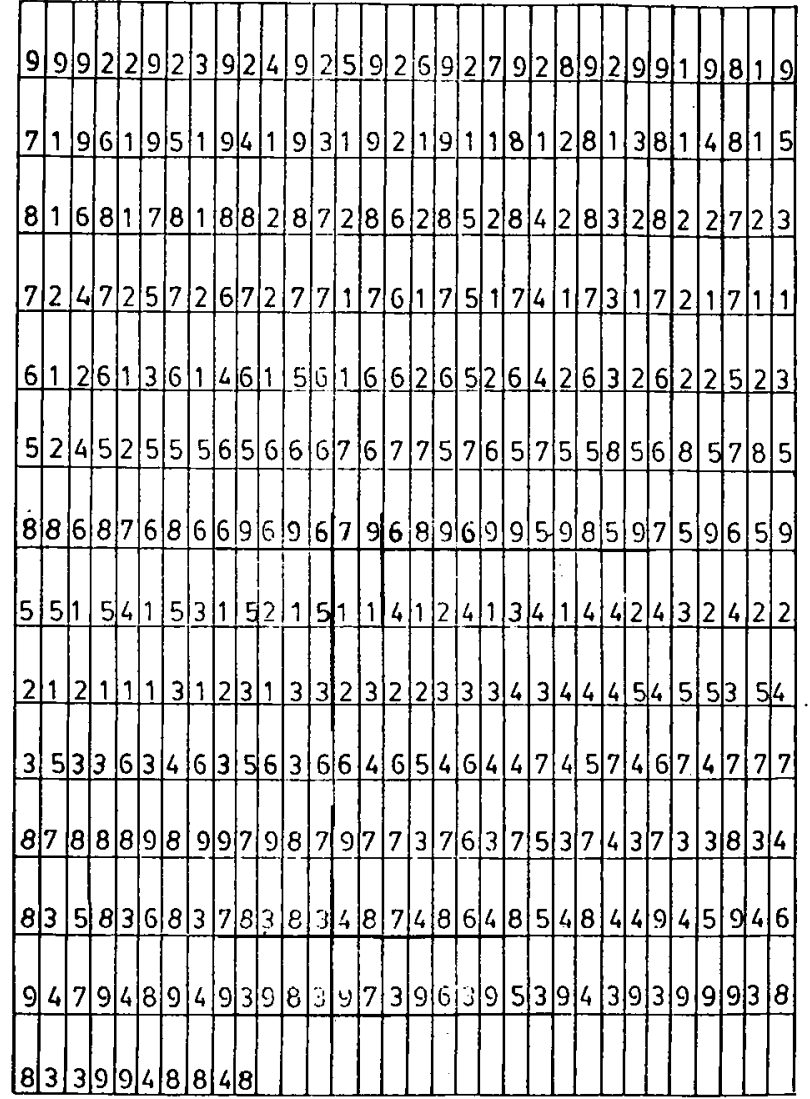

Fig 1. The unique sequence of combinations of alternate row design.

competing population. The average competing advantage (or disadvantage) for the expression of genotype $j$ with the competiting genotype $k$ is defined as $C_{k j}$ and with the competing genotype $m$ is defined as $C_{j m}$ and the specific interacting effect of the $k$ and $m$ competing genotypes on $j$ is defined as $S_{k j m} ; r_{i}$ is the effect of 
Table II. Expectations in the analysis of variance for competitive effects for all the genotypes.

Replication
Genotypes
Within genotypes
Treatments
Avg Com (Unadj)
Specific
$G \times T$
$G \times C^{\prime}$
$G \times S$
Error (unadj)
Total

$$
\begin{gathered}
\quad(r-1) \\
(p-1) \\
p\left(p^{2}+p-2\right) / 2 \\
\left(p^{2}+p-2\right) / 2 \\
\quad(p-1) \\
p(p-1) / 2 \\
(p-1)\left(p^{2} / p-2\right) / 2 \\
\quad(p-1) 2 \\
p(p-1)^{2} \\
(r-1)\left(p^{3}+p^{2}-2\right) / 2 \\
{\left[r p^{2}(p+1)-2\right] / 2}
\end{gathered}
$$

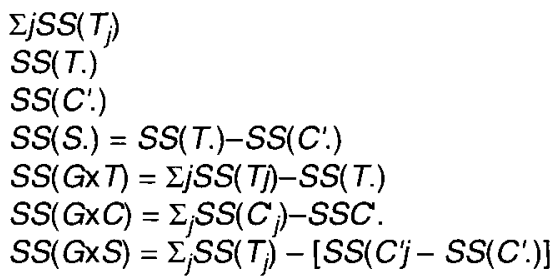

the th replication and $e_{i k j m}$ is the random error component. The expectations of variances due to different competitive effects of the genotypes are given in table II.

The expected sums of squares for the adjusted components in view of obtained adjusted error component (since the assumption that the possible order arrangements are equal eg $211=112$ causes an inflated experimental error) are given in table III.

Table III. Expected values for the adjusted components.

$\begin{array}{lcc}\text { Source } & d f & \text { Sums of squares } \\ & & \\ \text { Avg Comp adj }(C)(p-1) & S S(C .) \\ G \times C & (p-1) 2 & S S(G \times C)= \\ & & \Sigma_{j} S S\left(C_{j}\right)-S S(C .) \\ \text { Error adj } & (E)\left[(r-1)\left(p^{3}+p^{2}\right)-2\right] / 2(p-1) E^{\prime / p}\end{array}$

where:

$$
\begin{aligned}
& S S\left(T_{j}\right)=\Sigma_{k} \geq m\left(Y_{. j k m}\right)^{2 / r-2\left(Y_{. \mathrm{j} . .}\right)^{2} / r p(p+1)} \\
& S S(T .)=\Sigma_{k}\left(Y_{. \mathrm{km}}\right)^{2} / r p-2(Y \ldots) 2 / r p^{2}(p+1) \\
& S S(C .)=\Sigma_{k}\left(Y_{. k .}\right)^{2 / r p(p+2)-(Y \ldots .)^{2} / r p^{2}(p+2)} \\
& S S\left(C_{j}\right)=\Sigma_{k}\left(Y_{. j \mathrm{k} .}\right)^{2 / r}(p+2)-\left(Y_{\mathrm{j} . .}\right)^{2} / r p(p+2) \\
& Y_{. j \mathrm{~km}}=\Sigma_{i} Y_{\mathrm{ijkm}} ; Y_{. j \mathrm{k} .}=\Sigma_{\mathrm{m}} Y_{. j \mathrm{~km}} ; Y_{\mathrm{j} . .}=\Sigma_{\mathrm{i}} \Sigma_{k} \geq m Y_{\mathrm{ijm}} \\
& Y_{j k .}^{\prime}=\left(Y_{. j k .}-Y_{. j k k}\right) ; Y_{j . .}=\Sigma_{k} Y_{. j k}
\end{aligned}
$$

where:

$$
\begin{aligned}
& S S(C .)=\Sigma_{k}\left(Y_{. . k .}\right)^{2} / r p^{2}-(Y \ldots)^{2} / r p^{3} \\
& S S\left(C_{j}\right)=\Sigma_{k}\left(Y_{. \mathrm{jk} .}\right)^{2 / r p}-\left(\Sigma_{k} Y_{. \mathrm{jk}}\right)^{2 / r p^{2}}
\end{aligned}
$$

The competitive effect (adjusted) of the kth genotype measured by the th genotype is:

$$
C_{k j}=Y_{. k j} / r p-Y_{. j} / r p^{2}
$$

where: $C_{k j}$ measures the average increase or decrease of the th genotype when grown adjacent to a row of the $k$ th genotype. The average competitive effect (adjusted) of a genotype averaged for the rest of the genotypes is estimated as:

$$
C_{k .}=\Sigma_{j} C_{k} / p
$$

where: $C_{k}$ is the average increase or decrease of all the genotypes when bordered by the $k$ th genotype. The average competitive effects (adjusted) apply to a competing system where it is equally likely that any one the competing rows is any of the competing genotypes.

Expected values of a genotypes grown in nonbordered and bordered plots if each genotype occurred once within a block of a randomised block design are given respectively by:

$$
\begin{gathered}
Y_{k j}=\mu+G_{j}-2 C_{k j} \\
Y_{j j}=\mu+G_{j}-2 C_{j j}
\end{gathered}
$$

The average expected bias $\left(\Delta_{j}\right)$ for single row nonbordered plots in a randomised block design is:

$$
\Delta_{j}=-2 p G_{j j} /(p-1)
$$

where specific combination effects are ignored.

The error for testing $\left(C_{i j}-C_{. j}\right)$ comparison is $(p-1)^{3}$ $\sigma_{e}^{2 / r p^{3}}$.

\section{RESULTS AND DISCUSSION}

The analysis of variance was done for brown rust incidence recorded on first three dates (March 6, 
Table IV. Analysis of variance for the alternate row experiment for competitive effects on brown rust incidence pooled over all the genotypes.

\begin{tabular}{|c|c|c|c|c|c|}
\hline \multirow[t]{2}{*}{ Source } & & \multirow[t]{2}{*}{$d f$} & \multicolumn{3}{|c|}{ Mean square } \\
\hline & & & March 16 & March 25 & April 3 \\
\hline Replication & & 2 & 0.04 & 5.62 & 24.25 \\
\hline Genotypes & (G) & 8 & $314.07^{\star \star}$ & $6840.13^{\star \star}$ & $40395.75^{\star \star}$ \\
\hline Within genotypes & $(W)$ & 39 & $3.77^{\star \star}$ & $12.61^{\star \star}$ & $56.41^{\star \star}$ \\
\hline Treatments & $(T)$ & 44 & $4.58^{\star *}$ & $19.06^{\star \star}$ & $55.74^{\star \star}$ \\
\hline Average competition (unadjusted) & $\left(C^{\prime}\right)$ & 8 & $20.14^{\star *}$ & $102.92^{\star \star}$ & $161.06^{\star *}$ \\
\hline Specific combinations & $(S)$ & 36 & $1.12^{\star \star}$ & 0.43 & $32.33^{\star \star}$ \\
\hline$G \times T$ interaction & & 352 & 3.67 & $11.80^{\star \star}$ & $56.50^{\star \star}$ \\
\hline$G \times C^{\prime}$ interaction & & 64 & $11.10^{\star \star}$ & $49.48^{\star \star}$ & $147.57^{\star \star}$ \\
\hline$G \times S$ interaction & & 288 & $2.01^{\star *}$ & $3.43^{\star \star}$ & $36.26^{\star \star}$ \\
\hline Error (unadjusted) & & 808 & 0.29 & 3.01 & 3.41 \\
\hline Average competition (Adjusted) & & 8 & $12.89^{\star \star *}$ & $66.44^{\star \star}$ & $107.27^{\star \star}$ \\
\hline$G \times C$ (interaction) adj) & & 64 & $8.22^{\star \star}$ & $35.89^{\star *}$ & $116.35^{\star \star}$ \\
\hline
\end{tabular}

${ }^{\star},{ }^{\star \star}$ Significant at $P=0.05$ and 0.01 respectively.

March 25 and April 3 respectively) although a final record was made on April 12 (tables I à VI).

It could be observed that the genotypic component of variation was highly significant for brown rust incidence. The within-genotype component of variation was highly significant, which indicated that significant differences existed among genotypes for disease incidence. Significant $G \times$ $T$ interactions showed that the variation within a set of treatments was dependent on the genotypes involved. The genotypes IWP-19, IWP-72, IWP-124, IWP-127 and IWP-139 were in general resistant to natural brown rust infection showing very little or no infection irrespective of the nature of their neighbouring genotypes. The genotypes IWP-87-1, IWP-129, IWP-143 and Kalyansona, however, had varying levels of disease incidence based on their interactions with the genotypes in proximity and the date of observation. The unadjusted and adjusted average competitive effects were highly significant in all the dates. This indicated that performance of a line was significantly influenced by the genotypes bordering it. The significance of interaction effects suggested that both average as well as the specific competitive ability effects were genetically controlled.

On d 1 of observation (March 16), the onset of the rust was less dependent on the component effects as revealed by lower values of interaction effects. On the third date (April 3, ie, $120 \mathrm{~d}$ after sowing) however, all the components became im- portant. The general trend was that the competitive effects were of little importance when a virulent race of pathogen first appeared in the field. It attacked the susceptible plants regardless of whether they were bordered by resistant lines or not. The presence of plants which physically shielded the susceptible plants which allowed them to escape from infection as proposed by Luthra and Rao (1979), was not observed in the present study.

On the final date of observation on rust incidence (April 12), the components IWP-87-1 and Kalyansona were found to be heavily rusted regardless of the surrounding genotypes (table $\mathrm{V}$ ).

Hence, although the rate of rust development (r) when bordered by resistant lines may be less, if the rust sets in early in the season and if the crop growth period available for the rust to develop is long enough for many pathogen genera-

Table V. Summary of brown rust reaction on April 12 in 4 susceptible genotypes.

Genotype Brown rust reaction

IWP-87-1 Uniformly 100S

IWP-129 Around 50S

IWP-143 Varied from 60-80S

Kalyansona Uniformly 1005 
tions, the susceptible lines are more likely to be completely infected. If the rust had set in much earlier, then the possibilities of genotypes IWP129 and IWP-143 showing severe incidence cannot be ruled out unless some other aspects such as slow rusting are noted. Indications to support this conclusion are available from previous reports by Sitch and Whittington (1983) on powdery mildew in swede. After several pathogen generations and in spite of a period of disease restriction in the mixtures, the disease incidence in the mixture increased to the mean carrying capacity of the components. This might have happened due to the increase in disease severity on the susceptible host genotypes.

The classification of genotypes for brown rust reaction on the basis of $C_{k}$. values (ie, the competitive ability of the $k$ th genotype averaged over all the genotypes) for the 3 days of observation is given in table VI. It could be seen that Kalyansona was the genotype contributing maximum to the rust incidence.

The genotype $x$ competitive ability interaction component was significant for the rust intensity and hence, competitive effect of the th genotype when bordered by itself in comparison to when bordered by all the genotypes was computed and the results are given in table VII.

The incidence of brown rust on the th genotype when bordered by the same genotype in all the cases proved to be significantly positive and higher except in the case of IWP-129 on March 25 . On that date, disease incidence was higher in IWP-129 rows bordered by genotypes other than itself. Hence, it was suspected that it was getting the rust infection from some other susceptible border than itself. We attempted to find out which were the susceptible borders contributing to IWP129 's higher rust incidence and made comparisons among its combinations with Kalyansona, IWP-87-1 and IWP-143 (all 3 were highly contributory genotypes; see table VI) (table VIII).
The results revealed that IWP-129 when bordered by IWP-87-1 and Kalyansona had higher rust incidence than when bordered by all other genotypes.

However, in the case of IWP-143, the reverse was true as evidenced by the negative estimate. In other words, it is possible that race(s) for which IWP-87-1 and Kalyansona were susceptible also infected IWP-129. Kalyansona, a highly susceptible genotype caused a higher infection on IWP-129 compared to IWP-87-1.

The race infecting these 3 genotypes may be avirulent on IWP-143 and thus being resistant to this race, it might have restricted the development on IWP-129. However, IWP-143 might have been a host genotype for another race for which IWP-129 or possibly IWP-87-1 and Kalyansona were not susceptible. The results agree with the findings of Jeger et al (1983) in their study on Septoria nodorum in pure and mixtures of 2 spring wheat cultivars. They observed that the presence of a more resistant cultivar in the mixtures retarded the outward spread of the disease compared with the susceptible pure stand.

We computed the interaction estimates between Kalyansona (highly susceptible) and IWP. 87-1 (susceptible); results are provided in table IX.

The susceptible lines when bordered by themselves showed a higher incidence of rust. Similarly, they also showed a higher rust incidence when bordered by each other. This is indicated by the significant positive values obtained from the combinations IWP-87-1 vs Kalyansona and the latter $v s$ the former. The experimental mean of brown rust reactions of both Kalyansona and IWP-87-1 were less than values obtained when they were either bordered by themselves or when there was mutual bordering. Hence there seems to be an indication that the resistant lines might have lowered the magnitude of their mean values by reducing the infection rates of these

Table VI. The classification of genotypes for brown rust reaction on the basis of Ck values.

Genotype March 16 March 25 April 3 Remarks

\begin{tabular}{|c|c|c|c|c|}
\hline IWP-87-1 & 0.06 & 6.52 & 14.80 & Susceptible and highly contributory \\
\hline IWP-129 & -0.59 & -3.35 & 0.08 & Moderaltely resistant and highly contributory \\
\hline IWP-143 & -0.59 & -0.80 & 7.85 & Susceptible and very highly contributory \\
\hline Kalyansona & 3.90 & 16.59 & 37.34 & Highly susceptible and very highly contributory \\
\hline Other resistant genotypes & -0.59 & -3.59 & -12.06 & Non-contributory \\
\hline
\end{tabular}


Table VII. Comparison of auto- and mean infections $(\mathrm{Cjj}-\mathrm{C} . \mathrm{j})$ on 4 susceptible host genotypes on 3 dates of observation.

\begin{tabular}{|c|c|c|c|}
\hline \multirow[t]{2}{*}{ Genotype } & \multicolumn{3}{|c|}{$C i j-C . j$} \\
\hline & March $16^{+}$ & March $25^{++}$ & April $3^{+++}$ \\
\hline IWP-87-1 & $0.71^{\star \star}$ & $47.16^{\star \star}$ & $136.01^{* \star *}$ \\
\hline Kalyansona & $29.60^{\star \star}$ & $119.06^{\star \star}$ & $325.48^{\star \star}$ \\
\hline IWP-129 & 0.00 & $-29.77^{\star *}$ & $9.04^{\star \star}$ \\
\hline IWP-143 & 0.00 & $1.78^{\star *}$ & $85.18^{\star \star}$ \\
\hline
\end{tabular}

* ** Significant at $P=0.05$ and $P=0.01$ respectively; ${ }^{+}$error for March $16=0.006 ;^{++}$error for March $25=0.628 ;{ }^{+++}$error for April $3=0.078$.

Table VIII. The comparison of the incidence of brown rust on IWP-29 (March 25) when bordered by susceptible host genotypes and an apparently nonhost genotype.

$$
\text { Comparison } \quad C_{k 6}-\left(C_{k^{-}}-C_{6^{-}}\right) / 2+
$$

IWP-129 with IWP-87-1

IWP-129 with IWP-143

IWP-129 with Kalyasona

$20.33^{\star \star}$

$-13.01^{\star \star}$

$66.32^{\star \star}$

* Significant at $P=0.01 ;{ }^{\star \star}$ error $=0.628$

susceptible hosts. However, this is disputed by the records on the final date of observation (April 12 ; table V), by which time these susceptible genotypes were completely infected. The results support the views of Wolfe (1985) that if geno- types susceptible to the same pathogen race occur as neighbours, they will serve as the multiplication centres and thus hence enhance the rate of disease infection through the crop stand. Similar observations have been made with the development of powdery mildew in mixed stands of swede (Sitch and Whittington, 1983).

The results of the present study have shown that there was lack of evidence for the escape mechanism due to the shielding effect of the resistant lines during the initiation of the disease as proposed earlier by Luthra and Rao (1979). It is also clear that resistant genotypes may slow down the spread of the disease in the neighbouring susceptible genotypes by acting as physical barriers as described by Wolfe (1985) rather than completely protecting the latter from infection. Once the susceptible host genotypes are infected sufficiently early in the season, there are greater chances of these genotypes to be completely infected unless there are other probable mechanisms such as slow rusting or the resistance reduced by non-pathogenic spores (Wolfe, 1985).

The results of the experiment emphasise the need for testing the resistance of the component lines using the test of allelism for different $L r$ genes by artificial inoculations before synthesising the isogenic lines. Alternatively, the alternate row design or any appropriate design may also be used to study the progression of the disease in potential multiline cultivars in natural epiphytotic conditions. As such, a susceptible cultivar would interact with itself to give a higher incidence of disease and this effect may be confounded if there are 2 or more lines having susceptibility for the same race of the pathogen.

It is clear from tables $\mathrm{V}$ and VII that there may be at least 2 dominant races in the natural dis-

Table IX. Comparison of brown rust incidence on IWP-87-1 and Kalyansona mean values when bordered by themselves and while bordering each other.

Genotype combination

$C_{k j}-\left(C_{j}+C_{k}\right) / 2$

\begin{tabular}{ccc}
\hline March $17^{+}$ & March $25^{++}$ & April $3^{+++}$ \\
0.26 & $26.10^{\star \star}$ & $109.27^{\star \star}$ \\
$0.71^{\star \star}$ & $47.16^{\star \star}$ & $136.01^{\star \star}$ \\
$18.13^{\star \star}$ & $99.92^{\star \star}$ & $216.10^{\star \star}$ \\
$29.60^{\star \star}$ & $119.06^{\star \star}$ & $325.48^{\star \star}$ \\
12.59 & $80.75^{\star \star}$ & $225.59^{\star \star}$
\end{tabular}

** Significant at $P=0.01 ;{ }^{+}$error MSS $=0.006 ;{ }^{++}$error MSS $=0.628 ;{ }^{+++}$error MSS $=0.078$. 
ease infection during the season - the first infecting Kalyansona, IWP-87-1 and IWP-129 in common and the other infecting IWP-143. It is possible that the former rust race may cause more damage to this multiline if introduced into the Pantnagar environment.

It is pertinent to point out here that the results obtained in the present investigation using the alternate row design may not represent the true situation in a multiline variety. In a natural situation, the placement of all the 8 genotype plants is completely at random. In the alternate row design, barring the random allocation of different unique sequences of genotypes to different replications, the arrangements per se are fixed. Hence, the estimates of competitional variances tend to be biased in spite of adjusting the experimental error and average competitional effects for such bias. Secondly, in this design each host genotype can only have a maximum of 2 different neighbour genotypes. On the other hand, a component genotype in the multiline would have the chance of being surrounded by as many as 8 genotypes and consequently the interactions of genotypes for the disease expression in a particular host genotype also tend to be more complex. The alternate row design is a simple case of this situation since it corresponds to a mosaic of 9 genotypes each present 45 times. Because of this simplicity, it permits us to assess the influence of genotypes on the disease progression in a multine, particularly in cases where there are a greater number of component lines. Mundt and Brophy [1988] based on their computer simulation studies have strengthened the utility of alternate row design by stating that the culture of alternate rows of multiline components has the likelihood of better disease control than random mixtures.

\section{REFERENCES}

Buiel AM, Verhaar MA, Van Den Bosch F, Hoogkamer W, Zadoks JC (1989) Effect of cultivar mixture on the wave velocity of expanding stripe rust foci on winter wheat. Neth J Agric Sci 37(1), 75-78

Borlaug NE (1953) New approach to the breeding of wheat varieties to Puccinia graminis tritici. Phytopathology 43, 467

Borlaug NE (1957) The development and use of composite varieties based on the mechanical mixing of phenotypically similar lines developed through backcrossing. Proc 3rd Int Wheat Conf, 12-27
Borlaug NE (1958) The use of multilines or composite varieties to control airborne epidemic diseases of self-pollinated crop plants. Proc 1st Int Wheat Genet Symp, 12-27

Browning JA, Frey KJ (1960) Multiline cultivars as a means of disease control. Annu Rev Phytopathol 7, 355-382

Clifford PC (1975) Stable resistance to cereal diseases: Problems and progress. Rep Welsh Plant Breed Stat 1974, 101-103

Hanson WD, Brim CA, Kuel Hanson (1961) Design and analysis of competition studies with an application for field competition in soybean. Crop Sci 1 , 255-260

Jeger MJ, Jones DG, Griffiths E (1981) Disease progress of non-specialised fungal pathogens in intraspecific mixed stands of cereal cultivars. II. Experiments. Ann App/ Biol 98, 199-210

Jeger MJ, Jones DG, Griffiths E (1983) Disease spread of non-specialised fungal pathogens from inoculated point sources in intraspecific mixed stands of cereal cultivars. Ann App/ Biol 102(2), 237-244

Jensen NF (1952) Intra-varietal diversification in oat breeding. Agron J 44, 30-34

Luthra JK, Rao MV (1979) Escape mechanism operating in multiline and its significance in relation to leaf rust epidemics. Indian J Genet Plant Breed 39, 38-39

Mundt CC, Brophy CS (1988) Influence of number of host genotype units on the effectiveness of host mixtures for disease control: a modelling approach. Phytopathogy 78(8), 1087-1094

Mundt CC, Browning TA (1985) Development of crown rust epidemics in genetically diverse populations: effect of genotypic area. Phytopathology 75(5), 607-610

Mundt CC, Leonard KJ (1985) Effect of host genotype area on epidemic development of crown rust following focal and general inoculations of mixtures immune and susceptible oat plants. Phytopathology 75(10), 1141-1145

Mundt CC, Leonard KJ (1986) Analysis of factors affecting disease increase and spread in mixtures of immune and susceptible plants in computersimulated epidemics. Phytopathology 76, 832-840

Prakash KS, Gautam PL, Rao BP (1987) Studies on intergenotypic competition in a wheat multiline using alternate row design. J Plant Breed 98, 323-329

Prakash KS, Gautam PL, Rao BP (1990) Competitive interactions among eight near isogenic lines in a wheat multiline using a hill plot design. J Genet Breed 44, 91-96

Sitch L, Whittington WJ (1983) The effect of variety mixture on the development of swed powdery mildew. Plant Pathol 32, 41-46

Wolfe MS (1985) The current status and prospects of multiline cultivars and variety mixtures for disease resistance. Annu Rev Phytopathol 23, 251-273 